\title{
Modeling high-redshift galaxies: what can we learn from high and ultra-high resolution hydrodynamical simulations?
}

\author{
J. Devriendt ${ }^{1}$, A. Slyz ${ }^{1}$, L. Powell ${ }^{1}$, C. Pichon ${ }^{1,2}$ \& R. Teyssier ${ }^{3,4}$ \\ ${ }^{1}$ Astrophysics, University of Oxford, \\ Keble Road, Oxford OX1 3RH, UK \\ email: jeg@astro.ox.ac.uk \\ ${ }^{2}$ Institut d'Astrophysique de Paris, \\ $98^{\text {bis }}$ boulevard Arago, F-75014 Paris, France \\ ${ }^{3}$ Institute of Theoretical Physics, University of Zurich \\ Winterhurerstrasse 190, CH-8057 Zurich, Switzerland \\ ${ }^{4}$ Service d'Astrophysique, CEA Saclay \\ Bât. 141, F-91191 Gif-sur-Yvette, France
}

\begin{abstract}
We present results from a high resolution cosmological galaxy formation simulation called Mare Nostrum and a ultra-high resimulation of the first 500 million years of a single, Milky Way (MW) sized galaxy. Using the cosmological run, we measure UV luminosity functions and assess their sensitivity to both cosmological parameters and dust extinction. We find remarkably good agreement with the existing data over the redshift range $4<z<7$ provided we adopt the favoured cosmology (WMAP 5 year parameters) and a self-consistent treatment of the dust. Cranking up the resolution, we then study in detail a $z=9$ protogalaxy sitting at the intersection of cold gas filaments. This high-z MW progenitor grows a dense, rapidly spinning, thin disk which undergoes gravitational fragmention. Star formation in the resulting gas clumps rapidly turns them into globular clusters. A far reaching galactic wind develops, co-powered by the protogalaxy and its cohort of smaller companions populating the filaments. Despite such an impressive blow out, the smooth filamentary material is hardly affected at these redshifts.
\end{abstract}

Keywords. hydrodynamics, methods: numerical, galaxies: formation, galaxies: evolution

\section{Introduction}

Numerical galaxy formation simulations rely on modelling at least three basic components: collisionless dark matter and stars, and collisional gas. While techniques for evolving gravitating N-body systems representative of dark matter and stars, as well as for solving the gas dynamical Euler equations are advanced, additional physical ingredients present major modelling challenges. Once the gas within dark matter dominated gravitional potential wells cools, and condenses to the point of gravitational collapse, it is transformed into stars in an as yet unknown sequence of steps. These stars return gas, heavy elements, and energy back into their surroundings through feedback processes: massive, metal rich stars explode as supernovae. Not only is the theory of star formation a mystery, but it occurs on spatial scales several orders of magnitude smaller than the size of the host galaxy.

The work presented in this contribution tackles this complicated issue in two complementary ways. The first one consists in performing a statistical measure of global properties of marginally resolved (kpc resolution) galaxies by modelling a significant volume of the Universe. The second attacks the star formation and supernovae feedback 
physics through a tremendous increase of resolution to the subparsec level in the densest regions of a single galaxy. One of the motivations behind this strategy is to see whether simulations can describe accurately the bulk of the galaxy population and, at the same time, when run at the appropriate resolution, generate a galactic wind naturally, i.e. without a by hand implementation à la Springel \& Hernquist (2003). The results we present here focus on the high-z Universe.

\section{Numerical Simulations: the Set-up}

Both simulations make use of the Adaptive Mesh Refinement (AMR) code RAMSES (Teyssier 2002), which is run on different sets of initial conditions and using a different sub-grid physics model.

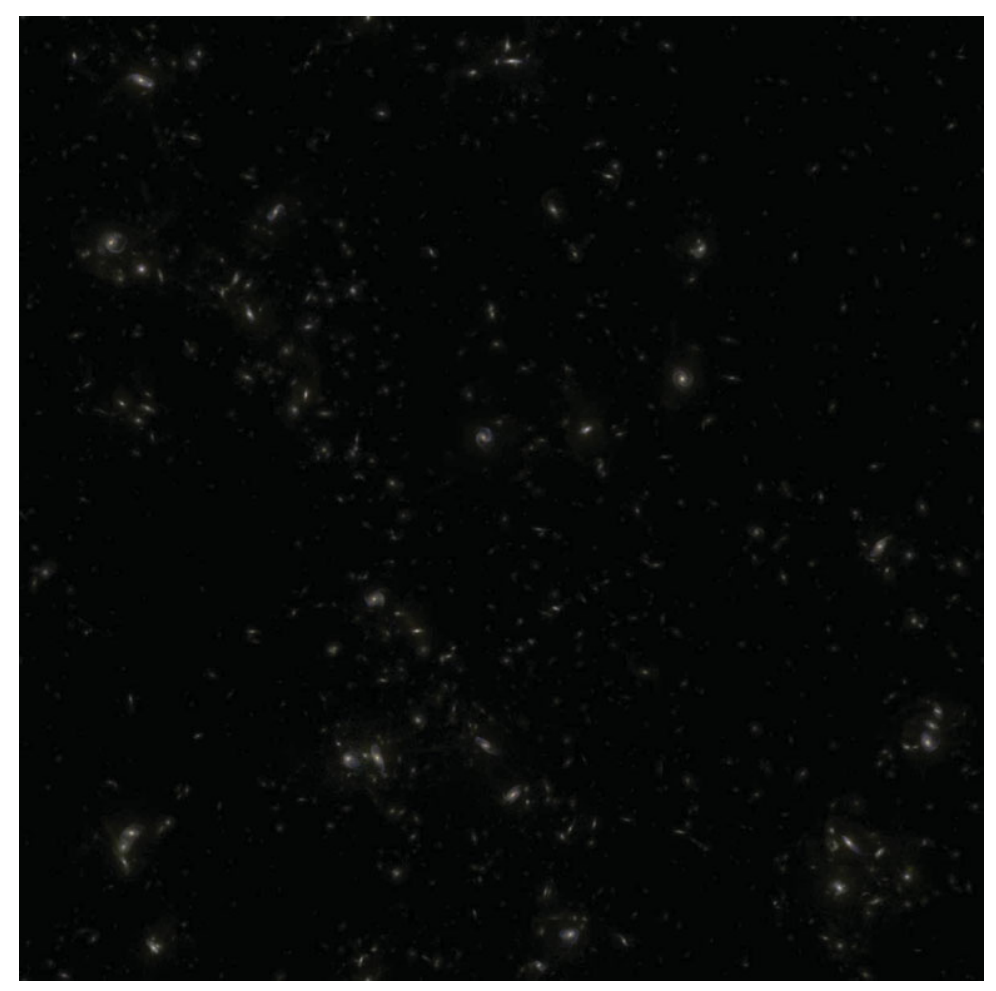

Figure 1. Projected composite image of a subfield of the Mare Nostrum simulation in true $\mathrm{R}, \mathrm{B}, \mathrm{V}$ coloras. See Fig. 2 for details on a smaller region.

\subsection{The Mare Nostrum Cosmological Simulation}

The evolution of a cubic cosmological comoving volume of $50 h^{-1} \mathrm{Mpc}$ on a side (see Fig. 1), containing $1024^{3}$ dark matter particles and an initial eulerian root grid of $1024^{3}$ gas cells is followed numerically. More specifically, we solve the coupled Vlasov-EulerPoisson equations in a $\Lambda \mathrm{CDM}$ concordance universe $\left(\Omega_{m}=0.3, \Omega_{\Lambda}=0.7, h=H_{0} /\right.$ $\left[100 \mathrm{~km} \mathrm{~s}^{-1} \mathrm{Mpc}^{-1}\right]=0.7, \sigma_{8}=0.9, n=1$, i.e. the WMAP 1 year best fit cosmology (Spergel et al. 2003)). This translates into a dark matter particle mass of $m_{p}=1.41 \times 10^{7}$ $\mathrm{M}_{\odot}$, and we use the AMR technique to keep our spatial resolution fixed at around $1 h^{-1} \mathrm{kpc}$ in physical coordinates, i.e. we progressively turn on more and more levels of refinement, until we reach 3 levels at $z=4$. In order to recast the results of our simulation for a different set of cosmological parameters (green curves on Fig. $3, \Omega_{m}=0.26, \Omega_{\Lambda}=$ 


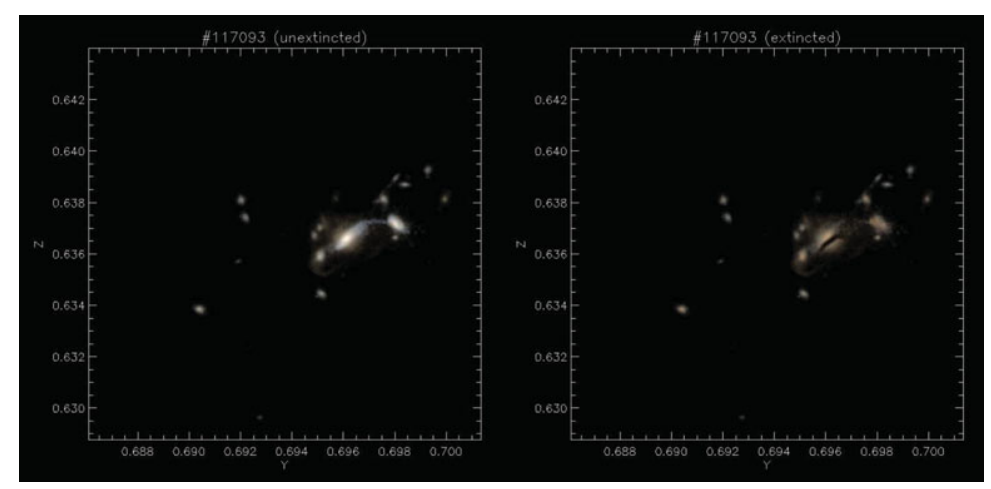

Figure 2. Zoom in on a smaller region of Fig. 1 neglecting dust extinction (left panel), or using the self-consistent extinction model described in the text (right panel). Note the redder colours overall and the presence of a well defined dust lane in the most massive galaxy in the bottom right panel, which highlights the non-homogeneous distribution of dust and stars in our virtual galaxies.

$0.74, h=H_{0} /\left[100 \mathrm{~km} \mathrm{~s}^{-1} \mathrm{Mpc}^{-1}\right]=0.72, \sigma_{8}=0.8, n=0.96$, i.e. the WMAP 5 year best fit cosmology (Dunkley et al. 2009)), we have used the Press-Schechter formalism to calculate the dark matter halo mass function at a given redshift in both cosmologies. Assuming an occupation number of one galaxy per dark matter halo, we then divide our luminosity functions by the ratio of the dark matter halo mass functions. Given the mass resolution of our simulation, we expect this correction to be quite accurate at higher redshifts (above $z=6$ or so) and to slowly degrade as more massive haloes begin to form and start hosting a larger number of galaxies on average. This explains most of the larger discrepancy between model and observed LFs at intermediate luminosities for $z=4$. Note that the correction remains valid at the faint and bright ends of the LF, as can be seen on the left panel of Fig. 3 .

\subsection{The Resimulation}

To perform a galaxy formation simulation with sub-parsec resolution in a cosmological context faithful to the currently accepted $\Lambda$ CDM paradigm, we apply a zoom technique whereby we focus our computing power on a subchunk of a larger volume of the Universe where a less expensive, low resolution dark matter only simulation predicts a galaxy will form. The host dark matter halo reaches a mass of $5 \times 10^{11} \mathrm{M}_{\odot}$ at redshift zero. The entire simulation takes place in a cubic periodic box of side length $9 h^{-1} \mathrm{Mpc}$. Out of this larger volume we carve out a subvolume of side length $\sim 3 h^{-1} \mathrm{Mpc}$ where we focus our resolution. A coarse $128^{3}$ grid covers the entire simulation volume, and in the subvolume we place an additional three nested grids, to give a minimal resolution of $1024^{3}$ in the subvolume throughout the course of the simulation. As the simulation proceeds we allow the grid in the subvolume to adaptively (de)refine so that the resolution in the densest regions is about 0.5 parsec (physical) at all times. The mass of dark matter particles in the high resolution subvolume is $\sim 5 \times 10^{4} \mathrm{M}_{\odot}$. With this dark matter resolution, our resimulations marginally resolve all potentially star forming halos contributing to the merger history of the galaxy under study.

\subsection{Cooling, Reionization, Star Formation and Supernova Feedback}

The cooling rates used in both simulations are metal dependent. However, in the cosmological simulation, gas is permitted to cool down to $10000 \mathrm{~K}$ only, whereas in the resimulation it reaches temperatures as low as a few tens of Kelvins. To model reionization, 
a spatially uniform ultraviolet background field that acts as a heating source is turned on instantaneously at a redshift of 8.5 (Haardt \& Madau 1996). Regarding the star formation implementation, we convert gas into stars in cells in which the gas density exceeds a threshold value $\left(\rho_{\text {thresh }} \sim 10^{-1}\right.$ atoms $\mathrm{cm}^{-3}$ in the Mare Nostrum run, $\rho_{\text {thresh }} \sim 10^{5}$ atoms $\mathrm{cm}^{-3}$ in the resimulation) at a rate proportional to the gas density in that cell, and inversely proportional to the free-fall time. The proportionality constant is the star formation efficiency and is taken to be $1 \%$. Of the gas mass removed to form a star, a fraction of it is locked up into a star permanently, and the other fraction is eventually ejected by a supernova. The latter produce metals which are advected as a passive scalar and incorporated into the cooling routines. In order to avoid artificial gas fragmentation on scales of the order of the Jeans length, a polytropic equation of state is enforced. Details of the star formation and supernova feedback implementation are given in Dubois $\&$ Teyssier (2008), and here we only remark on a key point. Contrary to the cosmological simulation where a star particle is very massive $\left(\sim 10^{6} \mathrm{M}_{\odot}\right)$, in our resimulations, the minimum star particle mass is $167 \mathrm{M}_{\odot}$, so that for a typical Initial Mass Function (IMF) (e.g. Salpeter) one expects one $10^{51}$ erg supernova explosion per star particle: we are resolving individual supernovae explosions.

\subsection{Dust Extinction}

We compute the spectral energy distribution (SED) of each our virtual galaxies in the Mare Nostrum, along the lines of the STARDust model (Devriendt et al. 1999). As we are interested in deriving a robust UV LFs, we need to estimate the fraction of stellar UV light absorbed by dust. Because a full modelling of the formation and evolution of the dust grain population is currently beyond our reach, we use two different sub-grid models to assess the impact of dust extinction on our results. First, as a sanity check, we adopt the most widespread method in the literature, which assumes a universal shape for the extinction curve (Calzetti et al. 1994), and then fits its normalization, E(B-V), in order to achieve the best possible match to the observational luminosity function. This means that the same average extinction is assigned to every galaxy, in contradiction with our empirical knowledge of the local Universe, where the value of the extinction is correlated to the UV magnitude (Heckman et al. 2005). However, invoking luminosity dependent extinction can be seen as a cheat, as it is equivalent to hiding the discrepancy between simulated and observed LFs in the extinction law. As a more ambitious alternative, we therefore measure the distribution of gas and metals on the line of sight towards each of the star particles in our simulated galaxies. This allows us to calculate the optical depth as a function of wavelength for each of these line of sight (see Fig. 1), based on the empirical model of Guiderdoni \& Rocca-Volmerange (1987). More specifically, as in this model, we assume that the optical depth, $\tau$, scales with the gas metallicity, $Z_{g}$, and in proportion to the column density of hydrogen along the line of sight, $N_{H}$, as:

$$
\tau(\lambda)=\left(\frac{A_{\lambda}}{A_{V}}\right)_{Z_{\odot}}\left(\frac{Z_{g}}{Z_{\odot}}\right)^{s}\left(\frac{N_{H}}{2.1 \times 10^{21} \mathrm{at} \mathrm{cm}^{-2}}\right)
$$

where $\left(A_{\lambda} / A_{V}\right)_{Z_{\odot}}$ is the extinction curve for solar metallicity, which we take to be that of the Milky Way. Following these authors, we adopt a scaling with metallicity of $s=1.6$ for $\lambda \geqslant 2000 \AA$ and $s=1.35$ for $\lambda \leqslant 2000 \AA$. We then calculate the extinction for subsamples of galaxies in logarithmic bins of stellar mass (100 objects per bin), based on the actual distribution of gas and metals in each galaxy and averaging over lines of sight. The resulting distributions are then used to draw random extinctions for other galaxies in the stellar mass bin. The total number of galaxies in the simulation varies between 43000 and 86000 from $z=7$ to $z=4$. 


\section{High-z UV LFs: Observations vs Cosmological Simulation}
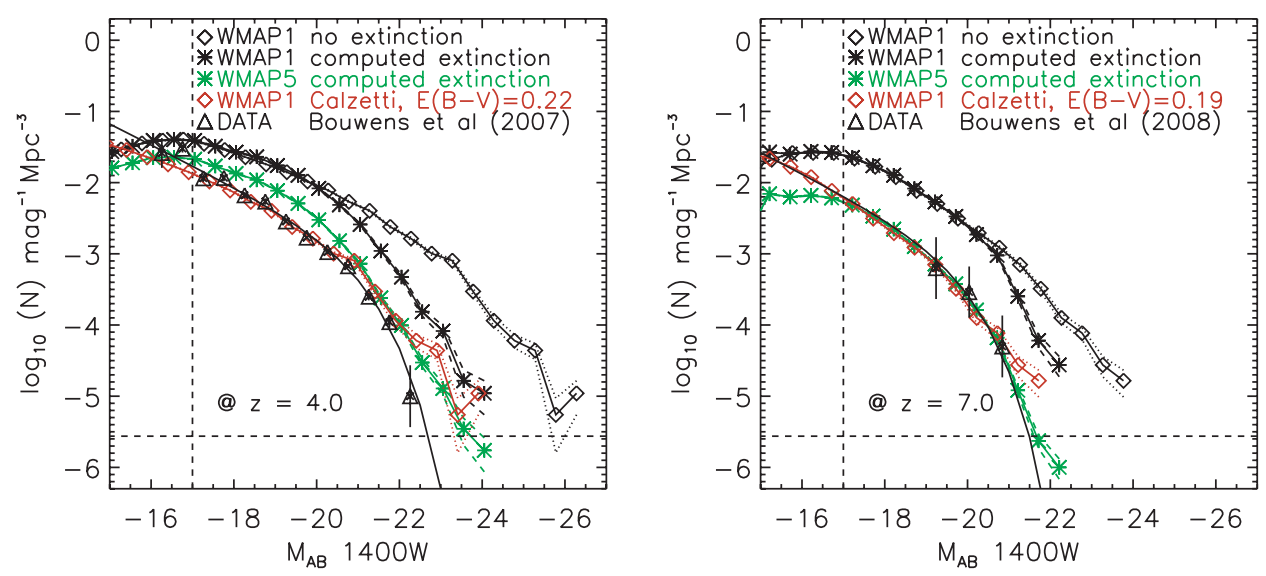

Figure 3. Luminosity functions (LFs) at $z=4$ and $z=7$ measured in the Mare Nostrum cosmological simulation described in the text. Vertical dashed lines indicate mass/luminosity resolution, while the horizontal ones define volume resolution. Black diamonds indicate non-extinguished WMAP 1 year cosmology LFs, black stars LFs extinguished with a dust content derived consistently from the simulation. Red diamond LFs stand for galaxies uniformly extinguished with a Calzetti law in a WMAP 1 year universe, and green stars for LFs extinguished as the black star ones but rescaled to the currently favoured WMAP 5 year cosmology. Black triangles and solid lines show data compiled by Bouwens and collaborators.

The two dust models produce LFs that match the observed one quite well within the whole range of magnitudes it covers, as observational error bars plotted on Fig. 3 only roughly estimate cosmic variance errors. Focussing on the self-consistent dust model, our simulated LFs at $z \sim 4$ are in respectable agreement with the available UV data. They robustly predict a faint-end slope with a value $\alpha \simeq 1.6$ compatible with the findings of Steidel and collaborators (Steidel et al. 1999 and follow up papers). Only our normalisation of $0.003 \mathrm{Mpc}^{-3}$ appears a bit high with respect to that measured in the data. However, this is the parameter of the Schechter function that is the most sensitive to our cosmological model correction. Our LFs also show quite an important amount of evolution between $z=4$ and $z=7$, with $\mathrm{M}_{\star}$, which varies from -20.9 to -19.8 respectively. This strong evolution is a direct consequence of the rapid decrease of the cosmic star formation rate density from $z=4$ to 7 in our simulation and is, to a large extent, unaffected by resolution effects. At $z>6$, recent analyses of the HUD and the GOODS fields (Bouwens et al. 2007, 2008) suggest a slightly steeper faint-end slope of $\alpha \simeq 1.74$ again in good agreement with our simulation as we find that the faint-end slope of our LF does steepen slowly with redshift. Finally, at the bright end of the LF, our two dust extinction models yield different results. The self-consistent extinction model has a more pronounced cut-off, which better matches the shape of a Schechter function. This feature stems from an intrinsically stronger dust attenuation that occurs in our most UV bright galaxies. We therefore predict that these UV monsters also are the most luminous infrared sources in the sky.

\section{Virtual Protogalaxies: a Filament-Wind Competition?}

The traditional story (White \& Rees 1978) of gas shock heating as it falls into a gravitational potential well before isotropically cooling onto a star-forming disk is being 


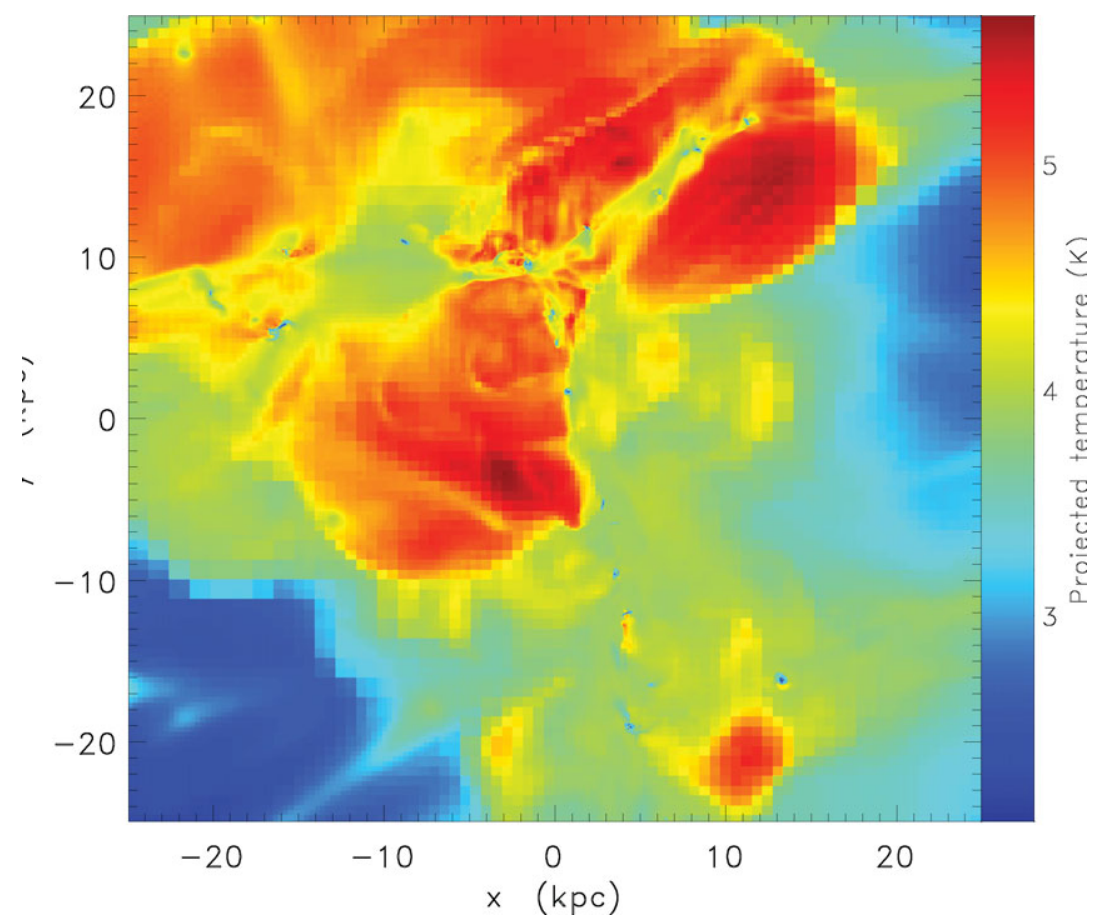

Figure 4. Projected temperature at $z=9$ of larger region around the main progenitor located at the epicentre of the hot, galactic wind and the intersection of 3 filaments dotted with cold substructures.
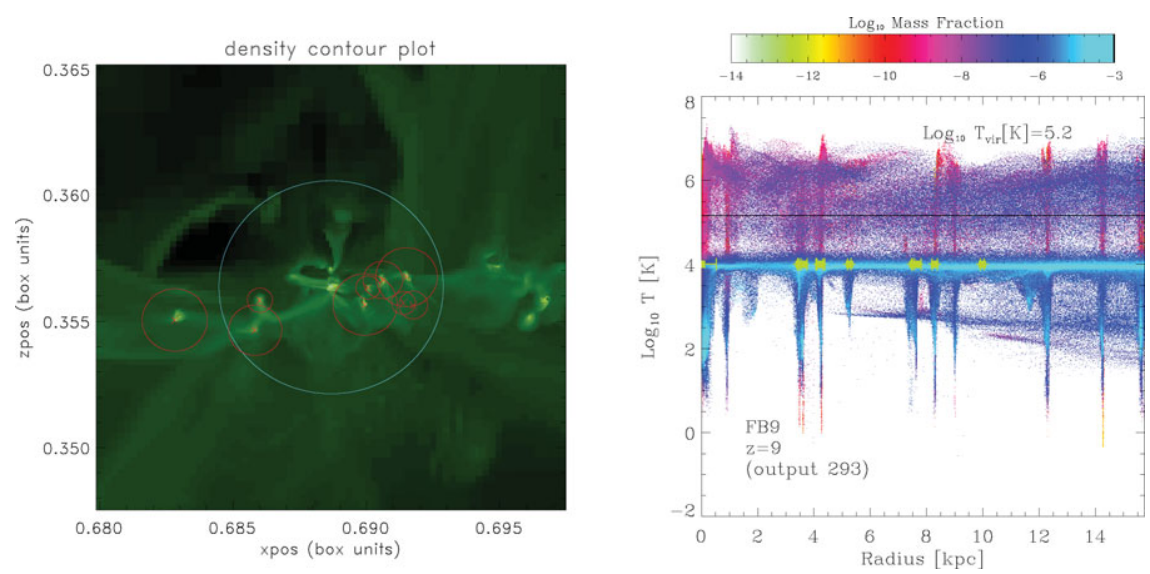

Figure 5. Left panel shows the dark matter particles (in blue) of the main halo at $z=9$ overplotted on the projected gas density (in green). Yellow points mark the dark matter particles within sub-halos. The circles centered on the main halo (turquoise) and its sub-halos (red) correspond to their virial radii respectively. Right panel shows the histogram of the mass-weighted temperature of the gas in radial bins centered on the main progenitor out to $\sim 3$ virial radii at $z=9$. Yellow-green points at $\mathrm{T}=10^{4} \mathrm{~K}$ denote the centers of sub-halos and the errors bars associated extend to $10 \%$ of their virial radius.

revisited. Recent work has emphasized the role played by filaments in transporting cold gas directly to the disk. Theoretical studies (Birnboim \& Dekel 2003, Dekel \& Birnboim 2006, Keres et al. 2005, Ocvirk et al. 2008, Brooks et al. 2009) stipulate a dark matter 
halo mass threshold $\left(\sim 510^{11} \mathrm{M}_{\odot}\right)$ dividing (a) low mass galaxies fed exclusively via filaments from (b) high-mass galaxies which accrete from a hot halo.

In contrast to previous similar studies, our high resolution simulation with supernova feedback finds a prominent, far reaching galactic wind (see Fig. 4) at high redshift around a protogalaxy sitting in a $\sim 5 \times 10^{9} \mathrm{M}_{\odot}$ dark matter halo. Therefore, despite being hosted by a halo well below the $\sim 5 \times 10^{11} \mathrm{M}_{\odot}$ mass threshold, our protogalaxy is immersed in hot - albeit non-gravitationally heated - gas. We attribute the origin of the hot wind to the overlap of supernova 'bubbles' emerging from the main protogalaxy and its neighbors (see left panel of Fig. 5). The spatially extended distribution of the sub-halos allows synchronized supernovae to reinforce themselves and build a sizeable wind. This scenario is corroborated by a histogram (right panel of Fig. 5) of the temperature of gas as a function of radius measured from the center of the main galaxy. Gas hotter than the virial temperature of the main halo exists at all radii probed in this figure (i.e. out to $3 \mathrm{r}_{\text {vir }}$ ). Spikes to both low and high temperatures coincide both with the main halo $(r=0)$ and the sub-halos visualized in the left panel of Fig. 5. Measurements carried out at larger radii reveal that the hot gas reaches $\sim 6 \mathrm{r}_{\mathrm{vir}}$ at this redshift. This is well-beyond the $\sim$ one $\mathrm{r}_{\mathrm{vir}}$ set down by the theory of gas shock-heated during accretion. Even though images of the gas density within the virial radius of the main progenitor show that the filaments streaming towards the central disk are perturbed by this violent supernova feedback, the measured accretion rates are hardly affected. Regardless of the presence of the wind, the total mass inflow rate has a value of approximately a few $\mathrm{M}_{\odot} \mathrm{yr}^{-1}$ at all radii measured out to the virial radius and is dominated by contributions from filaments which we define as all gas with temperature $\mathrm{T} \leqslant 2 \times 10^{4} \mathrm{~K}$ and densities $0.1 \leqslant \rho \leqslant 10$ atom $/ \mathrm{cm}^{3}$. Star formation is fuelled continuously.
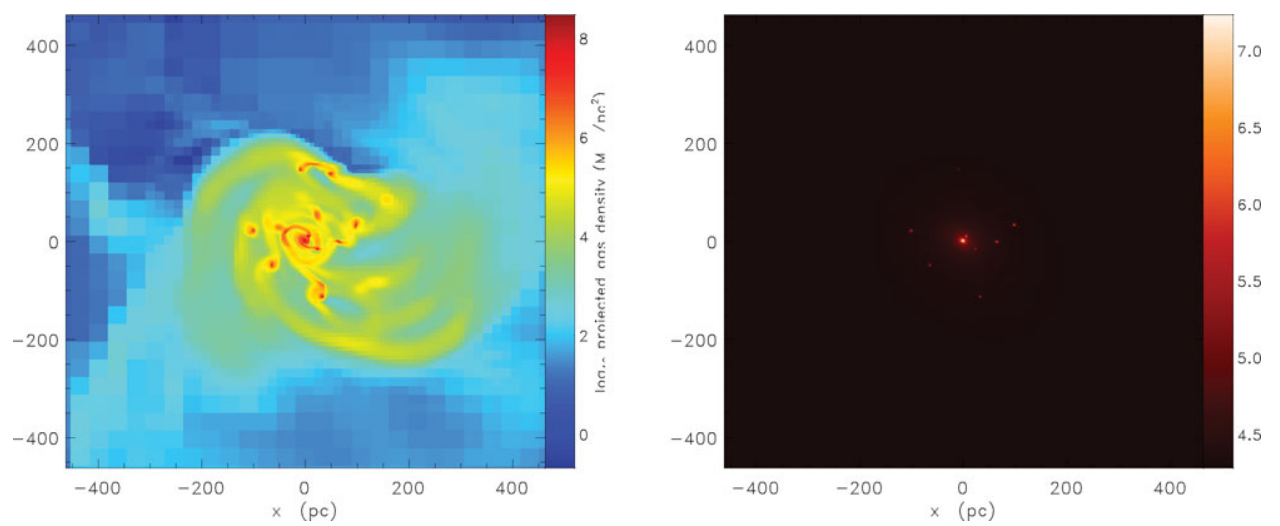

Figure 6. Projected gas density (left) and stellar density (right) of the main progenitor within the inner 10 percent of its virial radius at redshift 9.5 .

\subsection{Disk fragmentation}

A close-up view of the inner $10 \%$ of the virial radius reveals a dense and thin (scale height of $\sim 5 \mathrm{pc})$ gaseous disk, rotating nearly as fast as the Milky Way $\left(\mathrm{v}_{\text {rot }} \sim 140 \mathrm{~km} / \mathrm{s}\right)$ even though it is about a hundredth of its size $\left(\mathrm{r}_{\text {disk }} \sim 400 \mathrm{pc}\right)$. Under these extreme conditions, the disk is gravitationally unstable and fragments into massive gas "clumps" (Fig. 6, left) which quickly collapse into stellar clusters (Fig. 6, right). At $z=9.5,9$ of these clusters are present in the disk, with truncation radii ranging from 4 to $20 \mathrm{pc}$ and masses spanning $3.7 \times 10^{4} \mathrm{M}_{\odot}$ to $7.9 \times 10^{6} \mathrm{M}_{\odot}$. The remaining "diffuse" stellar component has a mass of $\sim 5.9 \times 10^{8} \mathrm{M}_{\odot}$. Therefore the globular clusters enclose a 
mass between 0.006 and $1.3 \%$ of the more smoothly distributed stellar component. We estimate typical gas surface densities $(\Sigma)$ and gas velocity dispersions $(\sigma)$ with massaveraged quantities within cylindrical shells. At $\mathrm{r} \sim 200 \mathrm{pc}$, we measure $\Sigma \simeq 500 \mathrm{M}_{\odot}$ $\mathrm{pc}^{-2}$ and $\sigma \simeq 16 \mathrm{~km} \mathrm{~s}^{-1}$, giving rise to a Jeans mass $\sim 5 \times 10^{6} \mathrm{M}_{\odot}$ consistent with the stellar clump masses.

\section{Discussion and conclusions}

We have derived UV luminosity functions including a self-consistent post-treatment of the dust extinction in virtual galaxies. We have successfully compared these synthetic LFs to the observed ones from $z=4$ to $z=7$. Future wider surveys together with the advent of infrared and deep millimetre observations from JWST and ALMA respectively will give us a robust handle on where energy from star formation is really coming out at these redshifts. These measurements will confirm or invalidate our prediction that dust attenuation strongly correlates with UV luminosity, yielding low luminosity galaxies almost dust free at $z \sim 7$, and massive galaxies extinguished by 1.5 to 2 magnitudes. If our model is correct, the bulk of the galaxy population will essentially be UV bright with a few isolated monsters shining across the entire wavelength range.

We are running a high resolution resimulation of a galaxy that will eventually reach a mass of $\sim 5 \times 10^{11} \mathrm{M}_{\odot}$ at redshift 0 . In its infancy $(\mathrm{z} \sim 9)$, this galaxy forms a dense, thin, rapidly rotating disk creating extreme conditions for star formation. Indeed, simple estimates of the Jeans mass in the gaseous proto-disk give values compatible with the picture that they form globular clusters with masses ranging from a few $10^{4}$ to a few $10^{6}$ $\mathrm{M}_{\odot}$. The synchronized supernovae exploding within the high-z main protogalaxy and its immediate neighbors result in a wind centered on the main protogalaxy that reaches well beyond $\sim 6$ virial radii of the main dark matter halo at $z=9$. The cold filaments within the virial radius of the main protogalaxy appear to become more diffuse when the wind becomes more pervasive, but measurements of the cold gas accretion remain unchanged, suggesting that the wind does not affect substantially the amount of cold gas supply to the disk.

\section{Acknowledgements}

The cosmological simulation was run at the Barcelona Supercomputer Centre, Spain, as part of the Horizon project (http://www.projet-horizon.fr). The single galaxy resimulation was performed on the JADE supercomputer at the CINES, France. We thank the staff of both institutions for their enthousiastic support.

\section{References}

Birnboim, Y., \& Dekel, A. 2003, MNRAS, 345, 349

Bouwens, R., et al. 2007, ApJ, 670, 928

Bouwens, R., et al. 2008, ApJ, 686, 230

Brooks, A. M., Governato, F., Quinn, T., Brook, C. B., \& Wadsley, J. 2009, ApJ, 694, 396

Calzetti, D, Kinney, A., \& Storchi-Bergmann, T 1994, ApJ, 429, 582

Dekel, A. \& Birnboim, Y. 2006, MNRAS, 368, 2

Devriendt, J. E. G., Guiderdoni, B., \& Sadat, R. 1999, $A \& A$ A, 350, 381

Dubois, Y. \& Teyssier, R. 2008, A\&A, 477, 79

Dunkley, J., et al. 2009, ApJS, 180, 306

Guiderdoni, B. \& Rocca-Volmerange, B. 1987, A\&A, 186, 1

Haardt, F. \& Madau, P. 1996, ApJ, 461, 20

Heckman, T. et al. 2005, ApJ, 619, L35 
Keres, D., Katz, N., Weinberg, D. H., \& Davé R. 2005, MNRAS, 363, 2

Ocvirk, P., Pichon, C., \& Teyssier, R. 2008, MNRAS, 390, 1326

Spergel, D., et al. 2003, ApJS, 148, 175

Springel, V. \& Hernquist, L. 2003, MNRAS, 339, 289

Steidel, C., et al. 1999, ApJ, 519, 1

Teyssier, R. 2002, A\&SA, 385, 337

White, S. \& Rees, M. 1978, MNRAS, 183, 341 